\title{
1 Sympatric wren-warblers partition acoustic signal space and song perch height
}

2 Shivam S. Chitnis ${ }^{1}$, Samyuktha Rajan ${ }^{1}$ and Anand Krishnan ${ }^{1}$

3 1- Indian Institute of Science Education and Research (IISER) Pune, Pashan Road, Pune

$4 \quad 411008$, India.

5 2- Author for correspondence: anandk@iiserpune.ac.in

$6 \quad$ Running title: Acoustic signal partitioning in wren-warblers

7 Keywords: acoustic space, prinia, perch height, niche partitioning, sympatric, acoustic signals

9 Abstract

10 By evolving divergent acoustic signals, sympatric assemblages of animals may minimize

11 potentially costly masking interference. Acoustic signal space may be multidimensional, with

12 coexisting species also vocalizing from different regions of physical space. Here, we

13 demonstrate acoustic signal space partitioning in four sympatric species of wren-warbler

14 (Cisticolidae, Prinia), in an Indian dry deciduous scrub habitat. We find that the breeding songs

15 of wren-warblers are divergent from each other in multivariate parameter space, with only

16 minimal interspecific overlap. Partitioning of signal space exerts constraints on the intraspecific

17 diversity of acoustic signals, and each species exhibits different strategies to overcome these

18 constraints. Two species partition intraspecific signal space into multiple note types, whereas a

19 third exhibits intraspecific variation in repetition rate, thus supporting song diversity within a

20 constrained acoustic space. Finally, we find that the four species also partition song perch

21 heights, thus exhibiting separation along multiple axes of acoustic signal space. We hypothesize

22 that divergent song perch heights may be driven by competition for higher singing perches or

23 other ecological factors rather than signal propagation. Acoustic signal partitioning along

24 multiple axes may therefore, we propose, arise from a combination of diverse ecological 25 processes. 


\section{Introduction}

29 The diversity of animal acoustic signals is thought to have evolved under the competing 30 pressures of remaining conspicuous to conspecifics, and simultaneously minimizing the risk of

31 predation or erroneous recognition(Wilkins et al. 2013). The latter is particularly relevant in 32 sympatric groups of closely related species, where errors in species recognition are costly(Grant 33 and Grant 2010). Considerable research has therefore focused on how the signals of closely-

34 related species diverge(Henry and Wells 2010). In multispecies assemblages, interspecific

35 divergence may result in a pattern where each species occupies a "niche" in signal space that is

36 distinct from all others(Chek et al. 2003; Schmidt et al. 2013). For birds, which use acoustic

37 signals to defend territories and attract mates, divergent acoustic signals enable them to

38 communicate with minimal competitive or masking interference from heterospecifics(Luther

39 2009). Signal space partitioning may occur along multiple axes; competition for singing

40 perches(Krams 2001; Sprau et al. 2012), or constraints related to maximizing sound

41 transmission(Marten and Marler 1977; Nemeth et al. 2002; Barker and Mennill 2009) may result

42 in sympatric species also partitioning physical space (i.e. singing perches) within the

43 environment. This may further minimize acoustic overlap between them.

44 Partitioning acoustic signal space along multiple axes, including evolving divergent frequency45 time parameters and singing from different locations, imposes constraints on the intraspecific 46 signal space of each species. By evolving diverse note types, or varying the temporal pattern of 47 songs, individual species may further partition intraspecific signal space, thus employing 48 complex communication in spite of the constraints of the "acoustic niche". Signal space 49 partitioning has been studied extensively in frogs and crickets (Duellman and Pyles 1983; Chek 50 et al. 2003; Schmidt et al. 2013). In birds, which often possess highly diverse and complex 51 songs, frequency and time-partitioning may help minimize acoustic signal interference(Fleischer 52 et al. 1985; Brumm 2006; Luther 2008; Krishnan and Tamma 2016). Additionally, a few studies 
53 have found perch height segregation in forest birds, attributed to maximizing sound

54 propagation(Nemeth et al. 2002; Barker and Mennill 2009; Tobias et al. 2010), minimizing

55 predation risk or male-male competition(Krams 2001; Sprau et al. 2012). However, signal space

56 partitioning in birds along multiple axes remains relatively understudied. Because of the varied

57 note types in the learned songs of passerine birds, studying sympatric groups of related species

58 may help understand how they overcome the limitations of their signaling niche. Additionally,

59 sound propagates differently in more open scrub/grassland habitats(Boncoraglio and Saino

60 2007), which are still underrepresented in tropical bioacoustics studies, and it is thus important

61 to study the acoustic signal space of birds occupying these habitats as a comparison to forest

62 birds.

63 Here, we study signal space partitioning along the axes of signal parameter space and physical

64 singing space (perch height) in four sympatric wren-warblers (Cisticolidae, genus Prinia)

65 occupying a seasonally dry scrub-grassland habitat in Maharashtra, Western Peninsular India.

66 These four congeners breed during the Southwest monsoons, during which they utter

67 conspicuous breeding songs. All four species are some of the most abundant singing birds in

68 this habitat(Krishnan 2019), suggesting that they are likely to encounter significant interspecific

69 overlap across all axes of acoustic signal space. We utilized a focal recording approach to

70 describe inter- and intraspecific variation in Prinia acoustic signals, and also recorded the perch

71 heights at which birds sang their songs. Our study illustrates the diversity of strategies that may

72 be employed by closely related birds to minimize masking interference and competition for

73 signaling space.

75 Materials and Methods

$76 \quad$ Study site and species

77 We conducted this study at the Vetal Tekdi Biodiversity Park, a small remnant of dry Acacia-

78 Anogeissus scrub-grassland mosaic with scattered trees in Pune, Maharashtra, India(Nerlekar 
79 and Kulkarni 2015). Four sympatric species of wren-warbler or prinia (genus Prinia) occur within

80 this landscape, the Ashy ( $P$. socialis), the Grey-breasted ( $P$. hodgsonii), the Plain ( $P$. inornata),

81 and the Jungle (P.sylvatica) prinias (Figure 1). Our study was timed to coincide with the onset of

82 the breeding season, with recordings conducted in June and July 2018 during the early

83 monsoon season.

85 Recording

86 Our recordings were made during the morning peak in singing activity, between 6-10AM. First,

87 we located singing birds and recorded their vocalizations using a Sennheiser ME62 (Wedemark,

88 Germany) omnidirectional microphone connected to a Zoom H6 (Tokyo, Japan) handheld

89 recorder. Each recording began as soon as the bird was located, and continued until the bird left

90 the singing perch. We kept notes on each recording both in a notebook and verbally at the end

91 of each recording, and transcribed them into datasheets after sampling sessions were

92 concluded. After concluding a single recording, we estimated the song perch height (see below),

93 made notes on species, type of vocalization (calls versus song) to match sound files to

94 measurements, and then proceeded to locate another vocal bird. Our total sampling effort at the

95 end of data collection resulted in about 160 individual measurements totaling approximately 4.5-

965 hours of audio, from which we measured a total of 4615 song notes across species (P.socialis

$97 \mathrm{~N}=1799$, P.hodgsonii $\mathrm{N}=1356$, P.inornata $\mathrm{N}=652$ and P.sylvatica $\mathrm{N}=806$ ).

99 Analyses

100 Using Raven Pro 1.5 (Cornell Laboratory of Ornithology, Ithaca, NY, USA), we labelled song 101 and call notes of all four species (Hann window size 512 samples with overlap of 256 samples) 102 and calculated the following nine parameters for each note: note duration, $90 \%$ bandwidth, 103 center frequency, average entropy, average peak frequency, maximum and minimum peak 104 frequency, and the peak frequency at the start and end of the note (calculated using the peak 
105 frequency contour feature in Raven Pro). We were careful to exclude recordings with high

106 background noise to avoid confounding variability in measured parameters. To visualize the

107 signal parameter space occupied by Prinia acoustic signals, we performed a principal

108 components analysis on the correlation matrix of all parameters measured (combining call and

109 song notes, as defined in the literature)(Rasmussen and Anderton 2005), and ordinated the

110 points corresponding to song notes in principal component space. Further, to examine how

111 distinct the song notes of different species were from each other, we carried out a linear

112 discriminant analysis (LDA) on the note parameters of the breeding songs, using the

113 Classification Learner app in MATLAB (Mathworks Inc., Natick, MA, USA). This analysis trained

114 a linear discriminant model and assessed its accuracy at classifying notes to species using 10-

115 fold cross-validation. We additionally performed a separate LDA on call notes (452 total notes

116 across species) as well, to examine interspecific differences between them. To further

117 statistically test for partitioning of note parameter space (considering notes of the breeding

118 songs only), we additionally performed two types of randomization analysis to quantify whether

119 average interspecific Euclidean distance in PC space was greater than expected by random

120 chance (indicating less overlap). In the first, we generated random distributions for each PC

121 (Supplementary Figure 1) spanning the same range of values, and then sub-sampled each PC

122 at random to generate a 4-species "null community", where each species had the same sample

123 size as the four in our dataset. Additionally, we performed a second randomization by randomly

124 reshuffling data points across species within our existing dataset to obtain a second type of "null

125 community". For both methods, we then calculated the average interspecific distance for each of

12610000 such randomized "null communities", and measured the Z score of the observed average

127 interspecific distance with respect to this randomized distribution. We predicted that a

128 partitioned song space would lead to a greater interspecific distance than random chance, and

129 therefore a significantly positive Z score. Finally, we performed randomizations using the first

130 method for each species pair (6 in total), to examine whether each pair exhibited greater 
131 interspecific distance than expected by random chance. This would indicate that each species

132 occupied a unique region of multivariate signal parameter space.

133 To examine whether species possessed multiple song note types within intraspecific song

134 signal space, we first examined the notes visually to identify broad groupings within species.

135 Next, we performed hierarchical clustering using the cluster function in MATLAB to empirically

136 verify whether clusters obtained using the previous method were indeed well-separated. As a

137 final quantification of the accuracy of this method, we performed a third LDA on these clusters to

138 assess how distinct and well-separated they were from each other. For species with only a

139 single note type, we determined whether they instead varied the repetition rate of their signals,

140 by examining variation in the interval between notes (the time between the end of one note and

141 the beginning of the next).

Perch height measurements

144 We estimated song perch heights in the field after each focal recording. Because of the inherent 145 uncertainty in estimates of perch height taken in the field, we grouped song perches into four 146 broad categories: perches $<2 \mathrm{~m}$ (grass and lower branches of bushes), $2-5 \mathrm{~m}$ (tops of bushes),

$1475-10 m$ (small trees and lower branches of large trees), and $>10 m$ (tops of large trees). To 148 statistically test whether perch height distributions across species differed from chance, we used 149 a $\chi^{2}$ test against an expected distribution assuming no interspecific patterns in perch height.

\section{Results}

152 Prinia species exhibit partitioning in acoustic signal parameters

153 The first three principal components of nine acoustic parameters explained over $77 \%$ of the 154 variation in Prinia acoustic signals (both songs and calls) (Table 1). PC1 loaded moderately 155 positively on all frequency parameters and moderately negatively on bandwidth and note 156 duration, whereas PC2 loaded moderately to strongly on entropy, bandwidth, starting peak 
157 frequency and maximum peak frequency. PC3 loaded positively on entropy and starting peak

158 frequency, and moderately negatively on average peak frequency and maximum peak

159 frequency. Within three-dimensional PC space, the breeding songs of all four species occupied

160 distinct regions largely separate from each other (Figure 2A, Supplementary Video, sample 161 sizes below).

162 Linear Discriminant Analysis (LDA) further supported the observation of Prinia species 163 occupying divergent regions of song parameter space from each other (Figure 2B). Our linear 164 discriminant classifier could assign song notes to species with an overall accuracy of $94.2 \%$. 165 P.socialis $(\mathrm{N}=1799)$ had a correct classification rate of $94 \%$, P.hodgsonii $92 \%(\mathrm{~N}=1356)$, 166 P.inornata 96\% (N=652) and P.sylvatica 97\% (N=806). The most likely misclassification (only $1676 \%$ ) was of P.socialis notes being misclassified as P.hodgsonii, and an $8 \%$ probability of the converse. The high accuracy of the LDA model, and low misclassification rates further supports these four sympatric species occupying distinct regions of song signal space with little overlap.

170 A similar LDA analysis on calls, classified separately from song notes, also uncovers some 171 separation between species (Supplementary Figure 2). Although calls of P.inornata and 172 P.sylvatica exhibited $99 \%$ and $100 \%$ correct classification rates in the LDA model, and 173 P.socialis $86 \%$, P.hodgsonii exhibited only a 55\% correct classification rate, and was $45 \%$ likely 174 to be misclassified as P.socialis. Thus, although Prinia are very distinct in song parameter 175 space, the calls of at least two species exhibit similarity to each other.

176 Finally, the results of a randomization analysis in 9-dimensional PC space (Supplementary 177 Figure 1, see Methods) also supported partitioning in song frequency-time parameters. Average 178 interspecific distance was much greater $(Z=63.04, P<0.001$ using distributions fit to our original 179 dataset, $Z=7.386 * 10^{12}, P<0.001$ using randomly reassigned data points, see Methods) than 180 expected by random chance (a distribution of 10000 randomized "null communities"). 181 Additionally, each species pair (6 total pairs for 4 species) was more divergent in song than 182 expected by random chance (Supplementary Data). Thus, our data are consistent with 
sympatric Prinia partitioning their breeding songs (but not necessarily call notes), and with each species occupying a unique region of 9-dimensional song parameter space.

Interspecific differences in complexity of song repertoires

187 Partitioning of acoustic signal space exerts a constraint on intraspecific (within-species) signal 188 diversity. We found that Prinia species exhibited different patterns in note diversity within 189 intraspecific signal space. Using hierarchical clustering (see Methods), we identified 5 distinct 190 note clusters in intraspecific principal component space for P.socialis (Figure 3A), and 7 for 191 P.hodgsonii (Figure 3B). An LDA on these clusters further supports their distinctness from each 192 other. The five clusters of notes in P.socialis all had correct classification rates between $81.3 \%$ 193 and $100 \%$, whereas the seven note clusters for P.hodgsonii had correct classification rates 194 between $75.9 \%$ and $100 \%$ (Supplementary Figure 3). Preliminary examinations suggested that 195 song bouts in P.socialis consisted of repetitions of a particular note type, whereas P.hodgsonii 196 combined up to 4 note types in a single song bout (Supplementary Figure 4).

197 In contrast to the former two species, P.inornata and P.sylvatica both exhibited only a single 198 note type with no clear clustering within intraspecific song parameter space (Supplementary 199 Figure 3). We therefore investigated whether they exhibited intraspecific variation in the 200 temporal pattern of their songs i.e. the inter-syllable interval. Probability density functions of the 201 inter-syllable intervals revealed that the two species exhibit different strategies from each other 202 (Figure 3C). P.sylvatica generally exhibited longer intervals but also a wider probability density 203 distribution across all recorded vocalizations of this species, peaking between $150-200 \mathrm{~ms}$. This 204 is consistent with the presence of faster and slower songs, and considerable intraspecific 205 variation in syllable repetition rate. By contrast, P.inornata exhibited a narrow peak at 206 approximately $40 \mathrm{~ms}$, indicating that its single note type is repeated rapidly at a more 207 stereotyped rate, with relatively little variation in timing. 
Interspecific differences in song perch height

210 Finally, we examined interspecific patterns in song perch height to quantify whether Prinia

211 species segregated from each other along this axis in acoustic signal space. Each species

212 exhibited a distinct pattern in the use of song perches (Figure 4). $P$. inornata sang below $5 \mathrm{~m}$

213 from the ground almost $60 \%$ of the time, and only very rarely from trees, whereas P.sylvatica

214 sang almost entirely ( $>80 \%$ of the time) from trees, and only very rarely near the ground.

215 P.socialis and P.hodgsonii sang mostly from intermediate heights, with the former distributing

216 almost evenly from 2 to $>10 \mathrm{~m}$, whereas the latter sang most commonly between $5-10 \mathrm{~m}$ above

217 the ground. Interspecific patterns in song perch heights were significantly different from chance

$218\left(\chi^{2}\right.$ tests for categorical data: $\chi^{2}=34.03, d F=9, P<0.01$, see Supplementary Data for more

219 details). Thus, the species with the least complex repertoires (in terms of note diversity),

220 generally sang from the highest and lowest song perches, whereas the two species with the

221 greatest note diversity sang from intermediate heights within their scrub-grassland habitat.

\section{Discussion}

224 In summary, we uncover evidence that four sympatric passerine wren-warblers in a dry 225 deciduous habitat in India are not only divergent in song parameters, but also exhibit partitioning 226 in song perch height. This therefore results in a restricted intraspecific acoustic signal space 227 along multiple axes, within which each species exhibits different note repertoires and diversity in 228 communication signals. The two species that occupy the highest and lowest singing heights 229 exhibit the most note stereotypy, and vary their note repetition rates to different extents. The two 230 species occupying intermediate heights exhibit multiple different note types, which likely 231 increases intraspecific signal variation within a constrained acoustic signal space. Below, we 232 discuss how the patterns exhibited by these passerine birds are illustrative of the constraints 233 that ecology and signal propagation may exert on signaling behavior. 
Acoustic signal space partitioning and the diversity of communication strategies

236 Partitioning of acoustic signal parameters minimizes overlap and masking interference(Schmidt et al. 2013), but additionally exerts a constraint on intraspecific signal diversity. Many organisms exhibit partitioning in frequency-time parameters of acoustic signals, including frogs, crickets and barbets(Chek et al. 2003; Schmidt et al. 2013; Krishnan and Tamma 2016). Both frequency and temporal traits are important facilitators in separating competing sound streams from each

241 other. The breeding songs of Prinia are issued primarily during the monsoon breeding 242 season(Rasmussen and Anderton 2005). In the landscape of seasonally dry scrub-grassland 243 that characterizes large parts of Peninsular India, the four species studied here are some of the 244 most abundant and vocal birds(Krishnan 2019), and are broadly sympatric over much of their

245 range. Partitioning acoustic signal space is thus likely to be important in minimizing masking 246 interference between them.

247 However, in addition to staying separate from heterospecifics, conspecific individuals must also 248 modify their signals and signaling behavior in order to remain distinct from each other. A restricted acoustic signal space as a result of physical constraints and niche partitioning 250 amplifies this problem. Our data suggests that wren-warblers overcome the constraints of their 251 signal space on intraspecific signal diversity in different ways. P.inornata possesses only a 252 single note type, and appears to sing a relatively stereotyped song without much variation. 253 P.sylvatica, however, which also has a single note type, exhibits a broad range of repetition 254 rates, consistent with possessing "faster" and "slower" songs. Finally, P.hodgsonii and P.socialis 255 exhibit 7 and 5 note types respectively. These may correspond both to greater note diversity 256 within a bout (as in P.hodgsonii), different song types (potentially what we observe in P.socialis) 257 or to individual variation in notes, which requires further study involving banding birds. Our study 258 examines note diversity only at the population level; what we do demonstrate, however, is that 259 closely related bird species may evolve intraspecific signal diversity in multiple ways within the 260 constraints of their acoustic niche. 
261 Finally, Prinia species possess multiple call notes (including contact and alarm 262 calls)(Rasmussen and Anderton 2005), in addition to their breeding songs. When examining 263 interspecific differences in call notes, we find that at least two species ( $P$.socialis and 264 P.hodgsonii) may possess relatively similar call structures (unlike their songs which are very 265 divergent), with high misclassification rates in an LDA analysis (Supplementary Figure 2). This 266 may indicate that their calls exhibit some degree of convergence in acoustic structure, possibly 267 owing to conserved function (for instance, alarm calls)(Bradbury and Vehrencamp 2011), and is 268 a potentially interesting subject of future study.

270 Perch height differences and the propagation of acoustic signals

271 In addition to partitioning their acoustic signals along frequency and time axes, a number of 272 animal taxa are also known to segregate along a spatial axis by selecting different singing 273 locations(Hodl 1977; Diwakar and Balakrishnan 2007). In many animals, this may help reduce 274 interference effects, although other studies have pointed out that height differences may be less 275 effective in reducing masking interference, especially if the overall height of available perches is 276 low(Schmidt et al. 2013). Singing height is known to influence the distance of sound 277 propagation(Marten and Marler 1977), and forest birds with lower pitches and slower songs sing 278 closer to the ground where their signals propagate further(Nemeth et al. 2002). We find that the 279 four Prinia species sing at different song perch heights. Interestingly, the two species with the 280 fewest note types ( $P$. inornata and P.sylvatica) sing at the lowest and highest heights, 281 respectively, and $P$. inornata, which sings very close to the ground, has the faster-repeating 282 song of the two. The other two species, which sing multiple note types $(P$. socialis and 283 P.hodgsonii) occupy intermediate perch heights.

284 P.sylvatica, which consistently sings at the highest perches, also has the lowest average peak 285 frequency and longest duration of its song notes, but the highest bandwidth on average of any 286 of the four species (Table 2). This runs contrary to studies of birds from closed rainforest, where 
287 lower-pitched, slower songs are found closer to the forest floor(Nemeth et al. 2002). One 288 possible reason for this is that our study was conducted in relatively open grassy habitats as 289 opposed to the closed-canopy forests where other such studies were undertaken. It is possible 290 that in such conditions, the higher bandwidths and longer notes of P.sylvatica enable longer291 distance communication. The narrow-band, rapid songs of P.inornata may enable it to avoid 292 detection by predators when singing very close to the ground. Studies have demonstrated a link 293 between song perch selection and predation risk(Krams 2001). Field experiments may help 294 address these questions by calculating relative signal propagation distances at various heights. 295 An alternate possibility is that perch height partitioning is driven by other ecological factors. 296 P.sylvatica is the largest of the four species(Grimmett et al. 1998), and we have observed 297 aggression towards other Prinia by this species, as well as by P.socialis, the next species in 298 size. Perch height partitioning may simply result from competition for higher song perches in this open environment, with P.inornata being driven to lower perches (intraspecific competition has been observed in nightingales)(Sprau et al. 2012). In support of this, all four species sing at least occasionally from the highest perches (Figure 4). Our data overall are consistent with a multidimensional acoustic niche in scrub-grassland passerine birds, with partitioning along multiple axes including frequency-time parameter space and physical singing space. The 304 physical axis of song perch height is putatively related to an ecological dimension, i.e. 305 competition for song perches and its consequences on signal propagation. Thus, we 306 hypothesize that perch height partitioning by itself may not play a role in segregating the 307 acoustic signal space of wren-warblers. By studying four closely-related species from the same 308 genus, we also highlight the diversity of communication strategies employed by singing birds to 309 minimize masking overlap while simultaneously generating diverse and distinct communication 310 signals. 
313 We thank Deepak Barua for help with equipment, Raghav Rajan and his lab for feedback and

314 discussions, and Samira Agnihotri for comments on the data.

315

316

317

318 Funding

319 AK is funded by an INSPIRE Faculty Award from the Department of Science and Technology,

320 Government of India and an Early Career Research (ECR/2017/001527) Grant from the

321 Science and Engineering Research Board (SERB), Government of India.

322

323 References

324 Barker NKS, Mennill DJ. 2009. Song perch height in rufous-and-white wrens: Does behaviour

325 enhance effective communication in a tropical forest? Ethology. 115(9):897-904.

326 doi:10.1111/j.1439-0310.2009.01674.x.

327 Boncoraglio G, Saino N. 2007. Habitat structure and the evolution of bird song: A meta-analysis

328 of the evidence for the acoustic adaptation hypothesis. Funct Ecol. 21(1):134-142.

329 doi:10.1111/j.1365-2435.2006.01207.x.

330 Bradbury JW, Vehrencamp SL. 2011. Principles of Animal Communication. 2nd ed. Sinauer

331 Associates.

332 Brumm H. 2006. Signalling through acoustic windows: Nightingales avoid interspecific

333 competition by short-term adjustment of song timing. J Comp Physiol A Neuroethol Sensory,

334 Neural, Behav Physiol. 192:1279-1285. doi:10.1007/s00359-006-0158-x.

335 Chek AA, Bogart JP, Lougheed SC. 2003. Mating signal partitioning in multi-species

336 assemblages: A null model test using frogs. Ecol Lett. 6:235-247. doi:10.1046/j.1461-

337 0248.2003.00420.x.

338 Diwakar S, Balakrishnan R. 2007. Vertical stratification in an acoustically communicating 
ensiferan assemblage of a tropical evergreen forest in southern India. J Trop Ecol. 23(2007):479. doi:10.1017/S0266467407004208.

341 Duellman WE, Pyles R a. 1983. Acoustic Resource Partitioning in Anuran Communities.

342 Copeia. 1983(3):639-649. doi:10.2307/1444328.

343 Fleischer RC, Boarman WI, Cody ML. 1985. Asynchrony of song series in the Bewick's wren

344 and wrentit. Anim Behav. 33(1969):674-676. doi:10.1016/S0003-3472(85)80095-6.

345 Grant BR, Grant PR. 2010. Songs of Darwin's finches diverge when a new species enters the 346 community. Proc Natl Acad Sci. 107(47):20156-20163.

347 Grimmett R, Inskipp C, Inskipp T. 1998. Birds of the Indian Subcontinent. 1st Editio. A\&C Black.

348 Henry CS, Wells MM. 2010. Acoustic niche partitioning in two cryptic sibling species of

349 Chrysoperla green lacewings that must duet before mating. Anim Behav. 80(6):991-1003.

350 doi:10.1016/j.anbehav.2010.08.021.

351 Hodl W. 1977. Call differences and calling site segregation in anuran species from Central

352 Amazonian Floating meadows. Oecologia. 363:351-363.

353 Krams I. 2001. Perch selection by singing chaffinches: a better view of surroundings and the 354 risk of predation. Behav Ecol. 12(3):295-300. doi:10.1093/beheco/12.3.295.

355 Krishnan A. 2019. Acoustic community structure and seasonal turnover in tropical South Asian 356 birds. Behav Ecol. doi:10.1093/beheco/arz087.

357 Krishnan A, Tamma K. 2016. Divergent morphological and acoustic traits in sympatric 358 communities of Asian barbets. R Soc Open Sci. 3(8):160117. doi:10.1098/rsos.160117.

359 Luther D. 2009. The influence of the acoustic community on songs of birds in a neotropical rain 360 forest. Behav Ecol. 20(4):864-871. doi:10.1093/beheco/arp074.

361 Luther DA. 2008. Signaller: receiver coordination and the timing of communication in 362 Amazonian birds. Biol Lett. 4(6):651-654. doi:10.1098/rsbl.2008.0406.

363 Marten K, Marler P. 1977. Sound transmission and its significance for animal vocalization - I.

364 Temperate habitats. Behav Ecol Sociobiol. 2:271-290. doi:10.1007/BF00299740. 
365 Nemeth E, Winkler H, Dabelsteen T. 2002. Differential degradation of antbird songs in a

366 Neotropical rainforest: Adaptation to perch height? J Acoust Soc Am. 110(6):3263-3274.

367 doi:10.1121/1.1420385.

368 Nerlekar AN, Kulkarni DK. 2015. The Vetal Hills $\square$ : an Urban Wildscape in Peril. Taprobanica.

369 07(02):72-78.

370 Rasmussen PC, Anderton JC. 2005. Birds of South Asia: The Ripley Guide. Lynx Edicions,

371 Barcelona.

372 Schmidt AKD, Römer H, Riede K. 2013. Spectral niche segregation and community

373 organization in a tropical cricket assemblage. Behav Ecol. 24(October):470-480.

374 doi:10.1093/beheco/ars187.

375 Sprau P, Roth T, Naguib M, Amrhein V. 2012. Communication in the third dimension: Song

376 perch height of rivals affects singing response in nightingales. PLoS One. 7(3):1-6.

377 doi:10.1371/journal.pone.0032194.

378 Tobias JA, Aben J, Brumfield RT, Derryberry EP, Halfwerk W, Slabbekoorn H, Seddon N. 2010.

379 Song divergence by sensory drive in amazonian birds. Evolution (N Y). 64:2820-2839.

380 Wilkins MR, Seddon N, Safran RJ. 2013. Evolutionary divergence in acoustic signals: causes

381 and consequences. Trends Ecol Evol. 28(3):156-166. doi:10.1016/j.tree.2012.10.002.

Tables

\begin{tabular}{|l|l|l|l|l|l|l|l|l|l|}
\hline & PC1 & PC2 & PC3 & PC4 & PC5 & PC6 & PC7 & PC8 & PC9 \\
\hline $\begin{array}{l}\text { Average } \\
\text { entropy }\end{array}$ & -0.22 & 0.35 & 0.56 & -0.04 & 0.66 & -0.22 & -0.10 & -0.10 & -0.08 \\
\hline Bandwidth & -0.37 & 0.36 & -0.21 & -0.02 & 0.09 & 0.50 & -0.21 & 0.09 & 0.61 \\
\hline $\begin{array}{l}\text { Center } \\
\text { frequency }\end{array}$ & 0.41 & 0.26 & -0.15 & -0.19 & 0.14 & -0.23 & -0.14 & 0.78 & 0.04 \\
\hline
\end{tabular}




\begin{tabular}{|c|c|c|c|c|c|c|c|c|c|}
\hline Duration & -0.41 & 0.06 & -0.21 & 0.04 & 0.03 & -0.42 & 0.73 & 0.15 & 0.21 \\
\hline $\begin{array}{l}\text { Peak } \\
\text { frequency }\end{array}$ & 0.32 & 0.23 & -0.36 & -0.60 & 0.26 & 0.11 & 0.26 & -0.43 & -0.06 \\
\hline $\begin{array}{l}\text { Peak } \\
\text { frequency } \\
\text { Start }\end{array}$ & 0.24 & 0.44 & 0.49 & 0.05 & -0.36 & 0.37 & 0.47 & 0.10 & -0.05 \\
\hline $\begin{array}{l}\text { Peak } \\
\text { frequency } \\
\text { End }\end{array}$ & 0.33 & -0.07 & -0.23 & 0.66 & 0.52 & 0.27 & 0.23 & 0.00 & -0.04 \\
\hline $\begin{array}{l}\text { Peak } \\
\text { frequency } \\
\text { Max }\end{array}$ & -0.07 & 0.64 & -0.34 & 0.36 & -0.25 & -0.27 & -0.22 & -0.22 & -0.31 \\
\hline $\begin{array}{l}\text { Peak } \\
\text { frequency } \\
\text { Min }\end{array}$ & 0.44 & 0.03 & 0.17 & 0.16 & -0.11 & -0.40 & -0.07 & -0.32 & 0.69 \\
\hline Eigenvalue & 4.32 & 1.67 & 0.98 & 0.64 & 0.59 & 0.29 & 0.28 & 0.13 & 0.09 \\
\hline $\begin{array}{l}\% \\
\text { explained }\end{array}$ & 47.97 & 18.54 & 10.87 & 7.15 & 6.58 & 3.23 & 3.18 & 1.46 & 1.01 \\
\hline
\end{tabular}

386 Table 1: Results of principal components analysis on the correlation matrix of nine acoustic 387 parameters measured from Prinia acoustic signals.

\begin{tabular}{|l|l|l|l|l|l|l|}
\hline & Bandwidth & Peak & Peak & Peak & Peak & Peak \\
& & Frequency & frequency \\
& & frequency & frequency & frequency \\
& & End & Max & Min \\
\hline P.socialis & 2169.5 & 5137.6 & 4394.4 & 4996.1 & 6279.9 & 3648.3 \\
\hline
\end{tabular}




\begin{tabular}{|l|l|l|l|l|l|l|}
\hline P.hodgsonii & 1822.5 & 5584.9 & 5783.9 & 5073.3 & 6529.9 & 4549.5 \\
\hline P.sylvatica & 3193.5 & 4479.5 & 4350.9 & 3898.0 & 6957.7 & 2769.3 \\
\hline P.inornata & 2003.7 & 4810.2 & 4835.1 & 4791.9 & 5902.3 & 3686.0 \\
\hline
\end{tabular}

Table 2: Frequency parameters and bandwidth (in $\mathrm{Hz}$ ) of all four species. Values represent averages for each parameter for all measured notes.

$394 \quad$ Figure legends

395 Figure 1: Breeding song spectrograms of four sympatric Prinia wren-warblers from Pune, 396 Maharashtra.

Figure 2: A. Principal component space (PC1-3) of the breeding songs of four Prinia species. Each species occupies a distinct region in this three-dimensional space. B. Confusion matrix of 400 a linear discriminant classifier built in MATLAB using 9 acoustic parameters. Percentages in the 401 green boxes indicate the correct classification rates for each species, and pink boxes the 402 misclassification rates. Note the high degree of accuracy of LDA in assigning calls to species, 403 indicating that they are divergent in signal space.

405 Figure 3: A, B. Note types of P.socialis (A) and P.hodgsonii (B). The different colors indicate 406 distinct note types, recovered using hierarchical clustering on all 9 parameters and then mapped 407 back onto two-dimensional PC space to visually depict the relative positions of each note type. 408 C. Probability density plots of the time interval between successive notes for P.inornata (blue)

409 and P.sylvatica (orange), two species with only one note type. Note that P.inornata sings a more 
410 rapid song (lower intervals), but that the peak is narrower. The broader peak of P.sylvatica

411 suggests more intraspecific variation in repetition rate.

413 Figure 4: Stacked bar graphs depicting the distribution of song perch heights occupied by four

414 Prinia species.

416 Supplementary Figure 1: q-q plots showing fits of each principal component score (PC1-9) to

417 a normal distribution (straight line). Because the data along each PC approximately follow

418 normal distributions, we fit random normal distributions to each and randomly resampled these

419 to test for non-randomness of acoustic community structure. See Methods for more details.

420 Bottom right: Frequency histogram of average interspecific distance for 10000 randomized "null"

421 assemblages of species, using these randomly generated distributions. The observed

422 interspecific distance in acoustic space is much larger, indicating partitioning of acoustic signal

423 space.

424

425 Supplementary Figure 2: Confusion matrix indicating LDA misclassification rates for the calls 426 of Prinia species. Color schemes are the same as in Figure 2.

428 Supplementary Figure 3: Top row: Song note PC space plots for P.inornata and P.sylvatica; 429 all song notes fall into a single cluster. Bottom row: Confusion matrices indicating LDA 430 misclassification rates for the note types of P.socialis (left) and P.hodgsonii (right).

432 Supplementary Figure 4: Spectrograms of different note types in P.socialis (top), and 433 P.hodgsonii (bottom, white box). Note that song bouts in P.socialis consist of repetitions of a 434 single note type, whereas P.hodgsonii incorporates multiple note types within a single song 435 bout. 
bioRxiv preprint doi: https://doi.org/10.1101/754606; this version posted September 1, 2019. The copyright holder for this preprint (which was not certified by peer review) is the author/funder. All rights reserved. No reuse allowed without permission.
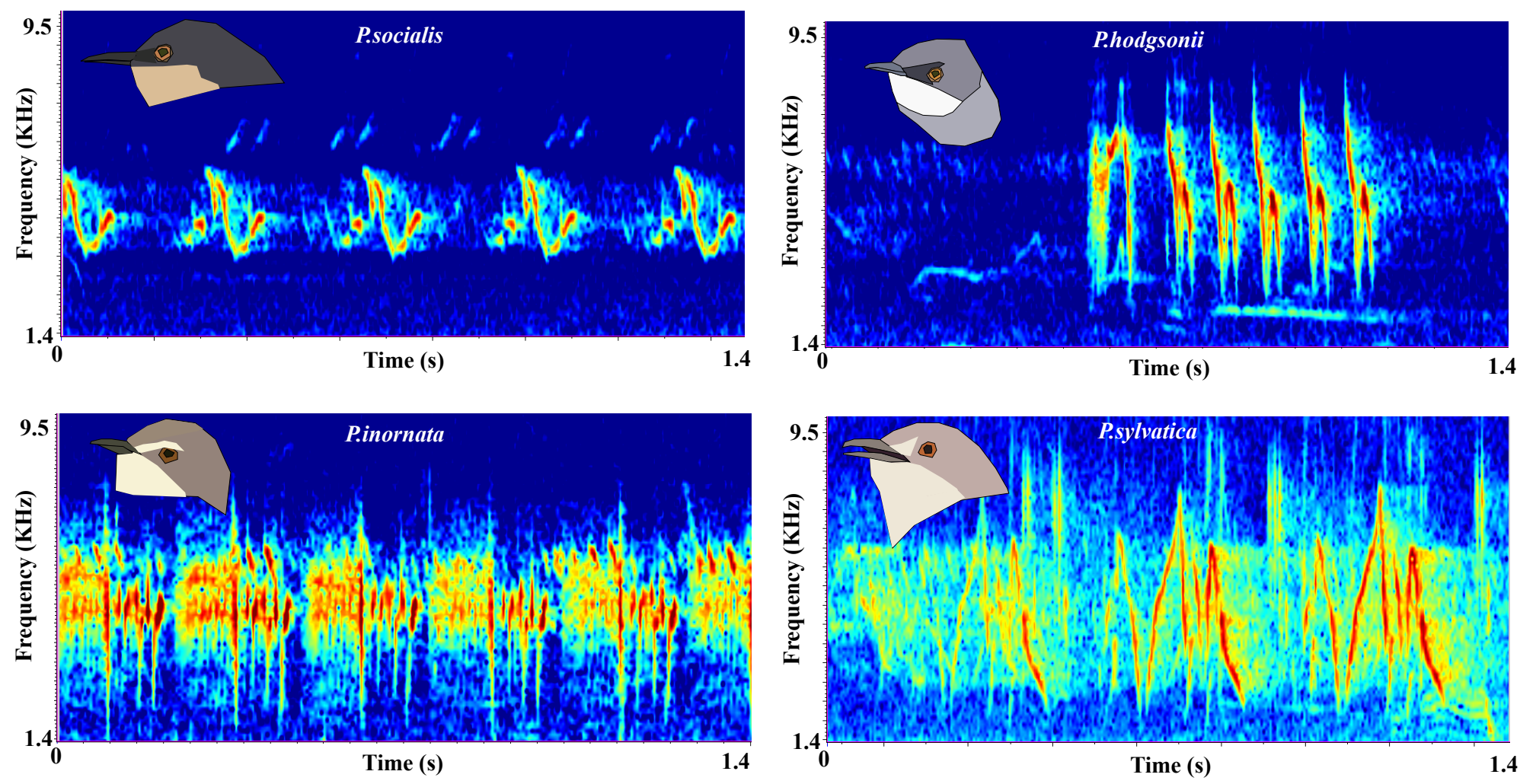
bioRxiv preprint doi: https://doi.org/10.1101/754606; this version posted September 1, 2019. The copyright holder for this preprint (which was

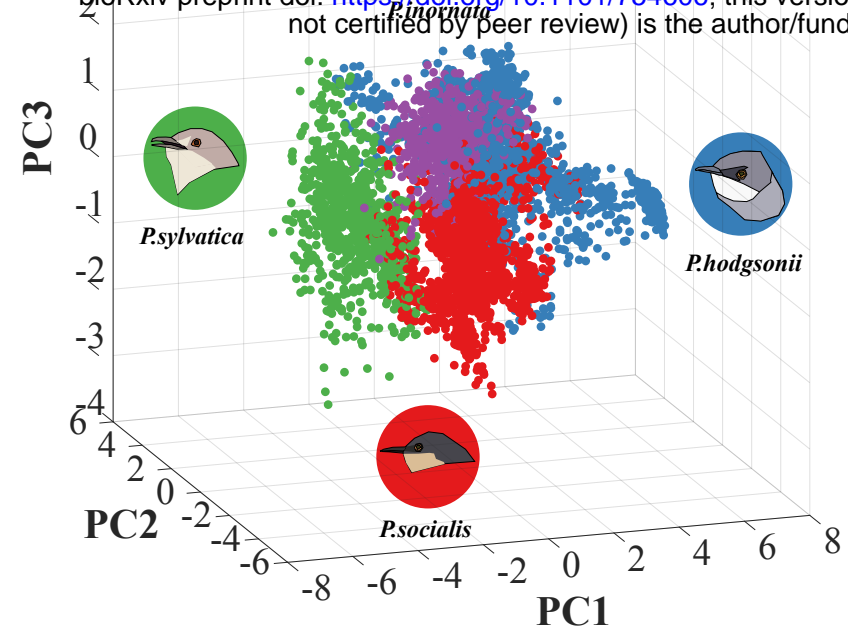

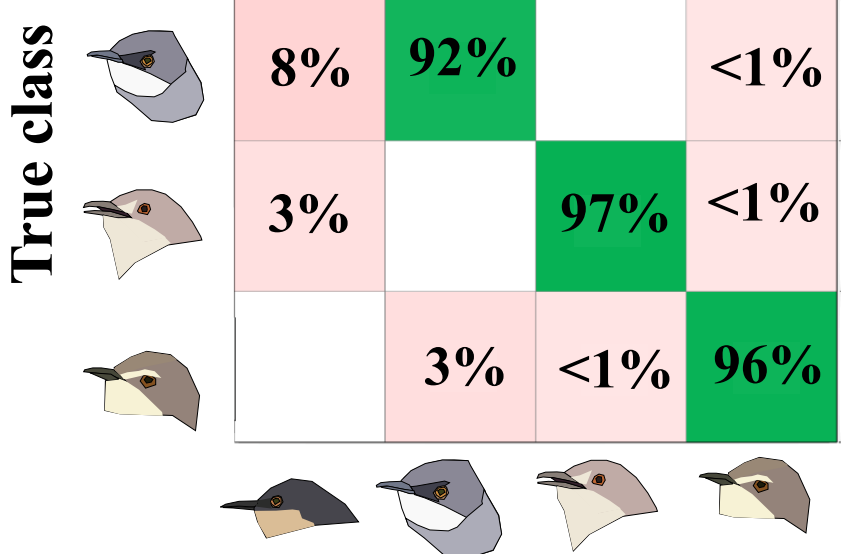

Predicted class 
A
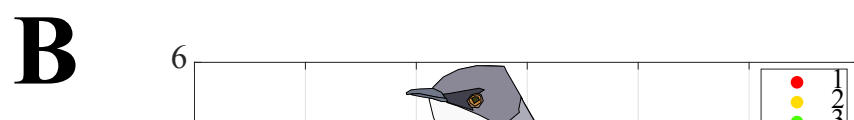
bioRxiv preprint doi: https://dol.org/T0.1101/754606,this version posted September 1, 2019. The copyright holder for this preprint (whith was
not certified by peer review) is the author/funder. All rights reseftued. No reuse allowed without permission.
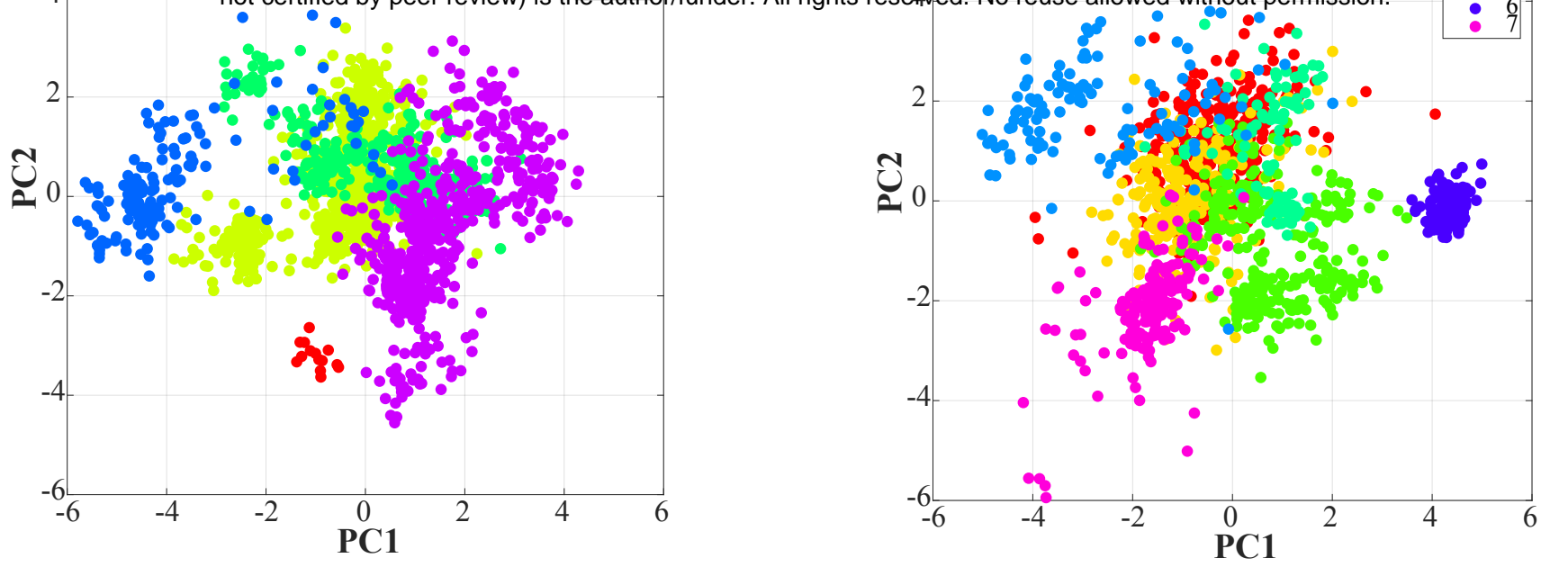

C

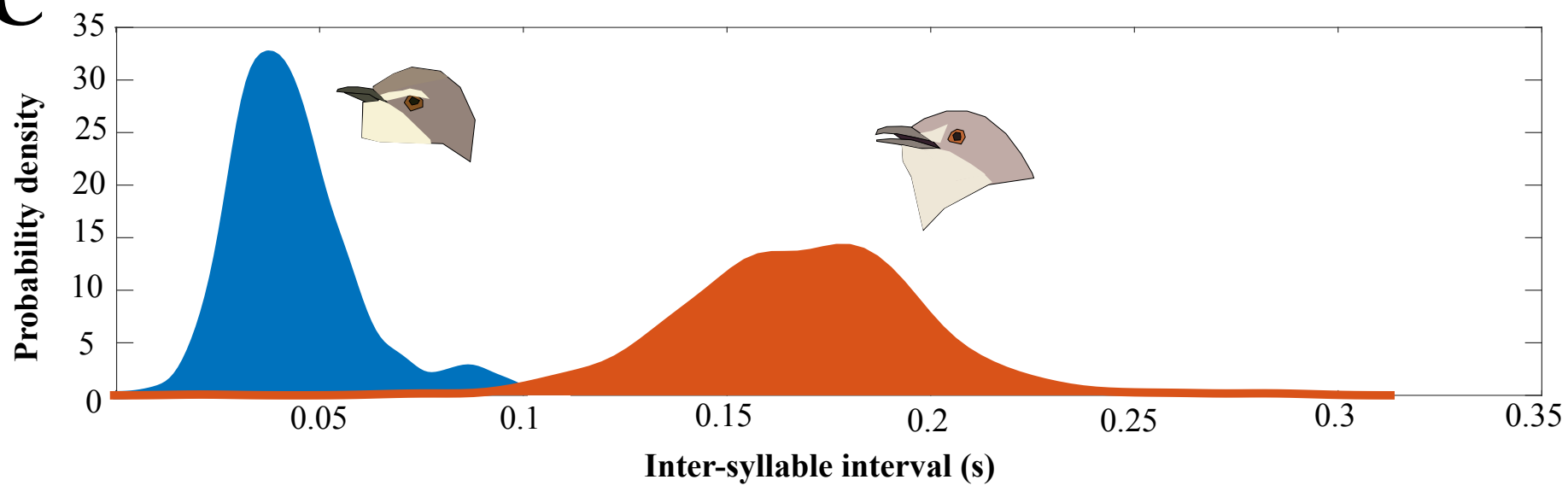


bioRxiv preprint doi: https://doi.org/10.1101/754606; this version posted September 1, 2019. The copyright holder for this preprint (which was not certified by peer review) is the author/funder. All rights reserved. No reuse allowed without permission.

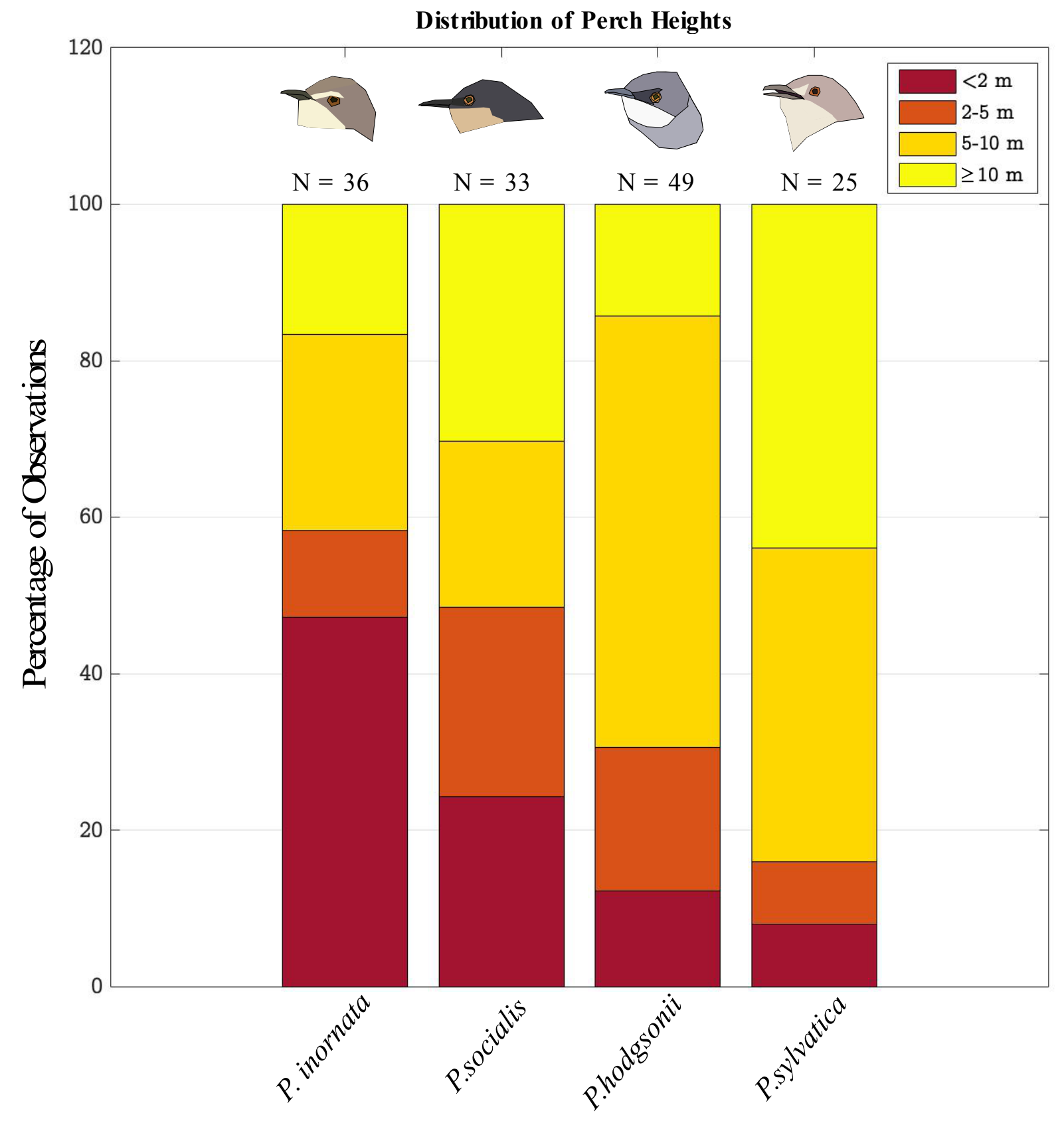

